\title{
Endometrial Histopathology in Patients with Laparoscopic Proven Salpingitis and HIV-1 Infection
}

\author{
Nelly R. Mugo, ${ }^{1}$ Julia Kiehlbauch, ${ }^{2,3,4}$ Nancy Kiviat, ${ }^{3}$ Rosemary Nguti, ${ }^{5}$ \\ Joseph W. Gichuhi, ${ }^{1,6}$ Walter E. Stamm, ${ }^{7}$ and Craig R. Cohen ${ }^{2,6,8}$ \\ ${ }^{1}$ Department of Obstetrics and Gynecology, Kenyatta National Hospital, P.O. Box 19865-00202, Nairobi, Kenya \\ ${ }^{2}$ Centre for Microbiology Research, Kenya Medical Research Institute, Nairobi, Kenya \\ ${ }^{3}$ Department of Pathology, Harborview Medical Center, UW Medicine Pathology, University of Washington, \\ P.O. Box 357470, Seattle, WA 98195-7470, USA \\ ${ }^{4}$ Maryland Department of Health and Mental Hygiene, 201 West Preston Street, Baltimore, MD 21201, USA \\ ${ }^{5}$ Department of Statistics, University of Nairobi, Nairobi, Kenya \\ ${ }^{6}$ Department of Obstetrics and Gynecology, University of Nairobi, School of Medicine, Kenyatta National Hospital, \\ P.O. Box 19676, Nairobi, Kenya \\ ${ }^{7}$ Department of Medicine, University of Washington, Seattle, WA 98195, USA \\ ${ }^{8}$ Department of Obstetrics, Gynecology and Reproductive Sciences, University of California, 50 Beale Street, Suite 1200, \\ San Francisco, CA 94105, USA
}

Correspondence should be addressed to Nelly R. Mugo, rwamba@csrtkenya.org

Received 18 April 2011; Revised 6 June 2011; Accepted 8 June 2011

Academic Editor: Thomas Cherpes

Copyright () 2011 Nelly R. Mugo et al. This is an open access article distributed under the Creative Commons Attribution License, which permits unrestricted use, distribution, and reproduction in any medium, provided the original work is properly cited.

Study Objective. To identify sensitive and specific histological criteria for endometritis in women with laparoscopically-confirmed acute salpingitis. Methods. Women, age 18-40 years of age presenting with complaints of lower abdominal pain $\leq 2$ weeks and no antibiotics use in past two weeks, were enrolled. They underwent clinical examination, screening for HIV; other sexually transmitted infections plus endometrial biopsy sampling for histopathology. Diagnostic laparoscopy confirmed the diagnosis of acute salpingitis. Controls were women undergoing tubal ligation and HIV-1 infected women asymptomatic for genital tract infection. Results. Of 125 women with laparoscopically-confirmed salpingitis, 38\% were HIV-1 seropositive. Nineteen HIV-1 negative controls were recruited. For the diagnosis of endometritis, $\geq 1$ plasma cells (PC) and $\geq 3$ polymorphonuclear lymphocytes (PMN) per HPF in the endometrium had a sensitivity of 74\% for HIV-1-seropositive, $63 \%$ for HIV-1-seronegative women with a specificity of $75 \%$ and positive predictive value of $85 \%$ regardless of HIV-1-infection for predicting moderate to severe salpingitis. For HIV-1-seronegative women with mild salpingitis, $\geq 1 \mathrm{PC}$ and $\geq 3 \mathrm{PMN}$ had a sensitivity of $16 \%$ and a PPV of $57 \%$. Conclusion. Endometrial histology, did not perform well as a surrogate marker for moderate to severe salpingitis, and failed as a surrogate marker for mild salpingitis.

\section{Introduction}

In Africa, pelvic inflammatory disease (PID) and its sequelae are a predominant cause of gynecologic morbidity $[1,2]$. These include tubal factor infertility, ectopic pregnancy, chronic pelvic pain, and recurrent pelvic infections $[3,4]$.

HIV-1 seroprevalence in women with PID is consistently 2-7 times greater than measured in matched populations without PID [5-7]; both infections are most commonly acquired through unprotected sexual activity. Prompt diagnosis and treatment of women with upper genital tract infections is important in reducing morbidity, but it is complicated by lack of a sensitive and specific clinical and laboratory diagnostic test. Laparoscopy is the gold standard for the diagnosis of salpingitis, but is not practical for routine clinical practice.

Endometrial histopathology is often used as a surrogate for upper genital tract infection. Kiviat et al. [8] evaluated 
women with clinical PID; evidence of endometritis as defined by $\geq 1$ plasma cell (PC) and $\geq 5$ polymorphonuclear lymphocytes (PMN) per high-powered field (hpf) was 92\% sensitive and $87 \%$ specific compared with visual findings of salpingitis determined by laparoscopy [8]. Using the same diagnostic criteria in a study of acute salpingitis in Kenya, plasma cell endometritis as defined by $\geq 1 \mathrm{PC} / \mathrm{hpf}$ was identified in $49 \%$ of women with salpingitis: this increased with disease severity and HIV-infection [7]. Studies on HIV1 -infected women have found an increased prevalence of plasma cell endometritis [9] even in the absence of clinical disease $[10,11]$.

Thus, we conducted this analysis to determine the optimum endometrial histopathological criteria for predicting salpingitis in a population with a high HIV-1 seroprevalence. We anticipate that these data will help to plan future clinical trials, increase the understanding of the pathogenesis of upper genital tract infection among HIV-1 infected women, and in certain circumstances provide a tool to confirm the clinical diagnosis of PID.

\section{Materials and Methods}

Study procedures have been previously detailed [12]. Briefly, between April 2000 and July 2002, women aged 18-40 admitted to Kenyatta National Hospital (KNH) acute gynecology ward with a complaint of lower abdominal/pelvic pain for 2 weeks or less plus one or more of the following signs or symptoms: temperature $\geq 38^{\circ} \mathrm{C}$, dysuria, and complaint of abnormal vaginal discharge were eligible for enrollment.

After induction of anesthesia, an endometrial biopsy was obtained with a Pipelle suction curette (Unimar, Inc., Wilton, Conn). At laparoscopy, samples from peritoneal fluid, tubal ostia, and pyosalpinx/tubo-ovarian abscess (TOA) were obtained for N. gonorrhoeae and C. trachomatis PCR. Using the Jacobson and Westrom criteria [13], the severity of acute salpingitis was graded as (1) mild (tubal erythema or edema, mobile tubes, and with or without spontaneous exudate), (2) moderate (marked tubal erythema and edema, limited tubal mobility, questionable or no tubal patency, and gross exudate), and (3) severe (pyosalpinx or TOA).

We enrolled two sets of controls. The first group included $\mathrm{HIV}-1$-seronegative women presenting to the KNH family planning clinic. Women desiring permanent sterilization underwent laparoscopic tubal ligation preceded by an endometrial biopsy obtained using a Pipelle suction curette. HIV-1-seropositive controls were enrolled from an HIV care and treatment clinic at the Center for Respiratory Disease Research at the Kenya Medical Research Institute. Subjects had no clinical evidence of PID. Enrollment and study procedures of the HIV-1-seropositive control group are detailed elsewhere [14]. After informed consent was obtained, an endometrial pipelle biopsy was obtained in the research clinic.

2.1. Laboratory Methods. Samples from the cervix, endometrium, fallopian tube, and abscess were examined by PCR (Roche Molecular Diagnostics, Pleasanton, Calif, USA) for
N. gonorrhoeae and C. trachomatis. Endometrial specimens were fixed in $10 \%$ buffered formalin, processed, and stained with hematoxylin, eosin, and methyl green pyronin. PMNs in glands and PCs in stroma were counted per high-power field. One pathologist (NK) who was blinded to the patients' diagnosis read the slides. Serum was tested for HIV antibodies by ELISA (Detect HIV, BioChem ImmunoSystems, Montreal, Canada) with positive results confirmed by a second ELISA (Recombigen, Cambridge Biotech, Ireland).

2.2. Data Analysis. Data were analyzed using SPSS for Windows 10.0 (SPSS Inc., Chicago, USA). Univariate analyses used chi-square and Fisher's exact tests for categorical data and Student's $t$-test for continuous variables. Logistic regression was done for multivariate analysis.

\section{Results}

3.1. Description of Study Population. One hundred and sixty women were enrolled with clinical PID, 140 (88\%) had laparoscopically confirmed salpingitis, 125 (89\%) of whom had an endometrial biopsy specimen: 56 (45\%) had mild, $31(25 \%)$ had moderate, and $38(30 \%)$ had severe disease based on laparoscopic criteria. Nineteen women had other diagnoses at laparoscopy including appendicular abscess $(n=2)$, endometriosis $(n=1)$, ovarian cyst $(n=$ $12)$, frozen pelvis $(n=1)$, pelvic tuberculosis $(n=1)$, cancer of the sigmoid volvulus with abscess $(n=1)$, and ovarian torsion $(n=1)$. Asymptomatic women $(n=20)$ desiring permanent sterilization underwent laparoscopic tubal ligation and served as HIV-1-negative controls. A single control subject had a sticky exudate emanating from the Fallopian tubes and was excluded from the analysis leaving 19 HIV-1-seronegative controls. Forty-five asymptomatic HIV1-seropositive controls were enrolled from an HIV care clinic; one woman had C. trachomatis detected.

Forty-eight $(38 \%)$ of the women with salpingitis were HIV-seropositive. Women with salpingitis were younger, less likely to be married, and less likely to have ever used contraception (Table 1). As expected, none of the HIV-1seronegative controls had signs or symptoms consistent with PID, and none were infected with $N$. gonorrhoeae or $C$. trachomatis. However, T. vaginalis was detected in a similar proportion of salpingitis cases (23\%) and HIV-seronegative controls (21\%) (Table 1).

\subsection{Factors Associated with Evaluable and Unevaluable Endometrial Histopathology. Of the 125 women with salpin- gitis, endometrial biopsies from 107 (86\%) were evaluated histological. Overall, 77 (72\%) were adequate for histological diagnosis. Inadequate biopsies corresponded to endometrial specimens demonstrating sloughing, frank pus, and lack of tissue. In general, more severe disease as demonstrated by higher clinical severity score (CSS) (15.5 versus 13, $P<.03)$ and severity of salpingitis based on laparoscopic findings $(P$-trend $<.04)$ was associated with unevaluable endometrial biopsy results (Table 2 ). Similarly, history of depomedroxyprogesterone acetate (DMPA) was associated}


TABle 1: Comparison of demographic, clinical history and signs, and laboratory findings for women laparoscopically diagnosed with salpingitis and women undergoing tubal ligation (controls).

\begin{tabular}{lcc}
\hline Variables & Salpingitis & HIV - /ve Controls \\
& $N=125$ & $N=19$ \\
\hline Demographics and clinical history & & \\
Age mean years (SD) & $27.8(5.5)$ & $34.3(4.1)$ \\
Education mean years (SD) & $8.7(2.9)$ & $8.2(2.5)$ \\
Marital status: & $31(25 \%)$ & 0 \\
$\quad$ Single & $70(56 \%)$ & $17(90 \%)$ \\
$\quad$ Married & $20(16 \%)$ & $2(10 \%)$ \\
$\quad$ Divorced/separated & & $16(84 \%)$ \\
Ever use of contraceptives: & $55(44 \%)$ & $15(80 \%)$ \\
$\quad$ Oral contraceptives & $40(32 \%)$ & $8(42 \%)$ \\
$\quad$ DMPA & $19(15 \%)$ & $3(16 \%)$ \\
$\quad$ IUD & $48(39 \%)$ & 0.001 \\
$\quad$ Condoms & & 0.12 \\
Clinical findings & $14(8) 32$ & 0.001 \\
Clinical severity score (CSS), median, (mode), range & & 0.001 \\
Laboratory findings & $48(38 \%)$ & 0.005 \\
HIV-1 & $23(18.4)$ & 0.04 \\
Gonorrhea and/or chlamydia & $23(19 \%)$ & 0 \\
Trichomonas vaginalis & $77(72 \%)$ & 0.00 \\
Adequate endometrial biopsy & & 0 \\
\hline
\end{tabular}

with an increased likelihood of obtaining an unevaluable biopsy $(P=.02)$. HIV-infected women were more likely to have an unevaluable endometrial biopsy (57\% versus $36 \%, P<.05)$ than HIV-uninfected women. Although not significant, participants with an inadequate endometrial histological specimen had a higher prevalence of gonorrhea compared to those with an adequate biopsy (23\% versus $12 \% P<0.23$ ) (Table 2).

In multivariate analysis, after controlling for factors found significant in univariate analysis, the use of DMPA at any time (adjusted OR $=3.1,95 \%$ CI 1.1-8.5), HIV-1 infection for women with mild $(\mathrm{AOR}=4.6,95 \% \mathrm{CI} 1.1-18.3)$ but not moderate salpingitis $(\mathrm{AOR}=0.89$, CI 0.15-5.3), or severe salpingitis $(\mathrm{AOR}=2.63$, CI $0.68-10.2)$ was associated with an increased odds of an unevaluable endometrial biopsy. In addition, 12 (63\%) of 19 specimens from HIV-1seronegative subjects were evaluable for histopathology.

3.3. Distribution of PMN and PC in the Endometrial Biopsy: Effect of HIV-1 Serostatus and Disease Severity. We reviewed the distribution of PMN and PC by HIV-1 serostatus and severity of salpingitis. Women with severe salpingitis regardless of HIV-1 serostatus had the highest frequency of PMN and PC per high-power field. Only two patients with HIV1 infection and salpingitis did not have PMN found in the endometrium. Although PMN density did not increase with severity of salpingitis among women with HIV-1 infection $(P$-trend $=.49)$, this association was significant for HIV-1uninfected women with salpingitis $(P$-trend $=.05)$. In contrast, the frequency of PCs increased with severity of salpingitis among those with HIV-1 infection $(P$-trend $=.04)$, but not among HIV-1 uninfected $(P$-trend $=.14)$. Furthermore, HIV-1 infection was associated with a higher frequency of $\mathrm{PCs} / \mathrm{hpf}(P$-trend $<.001)$, and presence of lymphoid follicles $(P<.04)$. Only $2(6 \%)$ of 34 HIV-1-infected women with salpingitis did not have any plasma cells present in the endometrium versus $23(41 \%)$ of $56 \mathrm{HIV}$-1-uninfected women with salpingitis.

3.4. Comparison of Endometrial Histopathology Findings and Salpingitis. We next set out to determine the sensitivity, specificity, and positive predictive value of four histopathologic criteria for diagnosis of endometritis in comparison to the laparoscopic diagnosis of salpingitis. The four rules evaluated included: (a) $\geq 3$ PMN and $\geq 1$ PC per highpower field, (b) $\geq 1 \mathrm{PMN}$ and $\geq 1 \mathrm{PC}$ per high-power field, (c) $\geq 1$ PMN per high-power field, and (d) $\geq 1$ PC per high-power field. Women with moderate and severe disease were grouped together and compared to women with mild salpingitis and to the two control groups. Table 3 outlines the comparison between the laparoscopic diagnosis for mild and moderate/severe salpingitis and the four histological rules stratified by HIV-1 serostatus. Because the diagnosis of the moderate and severe disease requires more objective evidence of tubal inflammation (e.g., pus from tubes, pyosalpinx, abscess, and fresh adhesions) than mild disease, we chose to gauge the sensitivity of each histological rule using laparoscopic evidence of moderate/severe salpingitis as the "gold standard." Rule "a", although less sensitive than rules "b" through " $d$ " for women with moderate/severe salpingitis (HIV-seropositive $=74 \%$ versus $63 \%$; HIV-seronegative; $93 \%$ versus $75 \%$ ), was the most specific, demonstrating 
TABle 2: Comparison of demographic, clinical history and signs, and laboratory findings for women laparoscopically diagnosed with salpingitis, with and without an endometrial biopsy adequate for histological evaluation.

\begin{tabular}{|c|c|c|c|}
\hline Variables & $\begin{array}{c}\text { Adequate biopsy } \\
N=77(72 \%)\end{array}$ & $\begin{array}{l}\text { Inadequate biopsy } \\
\qquad N=30(28 \%)\end{array}$ & $P$ value \\
\hline \multicolumn{4}{|l|}{ Demographics and history } \\
\hline Age mean (SD) & $27.8(5.5)$ & $27.9(6.1)$ & 0.9 \\
\hline Infertility $\geq 1$ year & $26(39 \%)$ & $3(13 \%)$ & 0.02 \\
\hline \multicolumn{4}{|l|}{ Ever use of contraceptive: } \\
\hline None & $56(51 \%)$ & $23(77 \%)$ & Ref \\
\hline Oral contraceptives & $36(47 \%)$ & $13(43 \%)$ & 0.75 \\
\hline DMPA & $18(23 \%)$ & $14(47 \%)$ & 0.02 \\
\hline Intrauterine device & $13(17 \%)$ & $3(10 \%)$ & 0.37 \\
\hline \multicolumn{4}{|l|}{ Symptoms } \\
\hline Abnormal menstruation & $16(21 \%)$ & $6(21 \%)$ & 1.0 \\
\hline \multicolumn{4}{|l|}{ Clinical examination findings } \\
\hline \multicolumn{4}{|c|}{ Clinical severity score, median (mode) range } \\
\hline Total clinical severity score & $13(8) 28$ & $15.5(4) 30$ & 0.03 \\
\hline \multicolumn{4}{|l|}{ Laparoscopic salpingitis severity } \\
\hline Mild & $32(42 \%)$ & $15(50 \%)$ & \\
\hline Moderate & $26(36 \%)$ & $3(10 \%)$ & \\
\hline Severe & $19(25 \%)$ & $12(40 \%)$ & 0.04 \\
\hline Pelvic Abscess & $13(17 \%)$ & $9(30 \%)$ & 0.15 \\
\hline \multicolumn{4}{|l|}{ Laboratory Findings } \\
\hline HIV-1 & $27(36 \%)$ & $17(57 \%)$ & 0.05 \\
\hline CD 4 count $<200 / \mu \mathrm{L}$ & $8(11 \%)$ & $7(23.3 \%)$ & 0.09 \\
\hline White cell count & $9.7(6.04)$ & $10.3(5.5)$ & 0.63 \\
\hline Lymphocytes (blood) \% & $26.9(13.4)$ & $19.1(7.8)$ & 0.001 \\
\hline Gonorrhea and/or chlamydia & $11(14 \%)$ & $8(27 \%)$ & 0.13 \\
\hline Gonorrhea & $9(12 \%)$ & $7(23 \%)$ & 0.23 \\
\hline Chlamydia & $3(4 \%)$ & $1(3 \%)$ & 0.9 \\
\hline Trichomonas vaginalis & $14(19 \%)$ & $6(21 \%)$ & 0.8 \\
\hline Bacterial vaginosis (Gram's stain) & $30(45 \%)$ & $16(62 \%)$ & 0.2 \\
\hline
\end{tabular}

endometritis in $25 \%$ and $7 \%$ of HIV-1-seronegative and HIV-1-seropositive controls, respectively, in comparison to $58 \%-67 \%$ and $38 \%-62 \%$ for rules "b" through " $\mathrm{d}$ ". Among the 19 women enrolled with a clinical diagnosis of PID, but who did not have salpingitis on laparoscopy, rule "a" had the least false positive, while rule "d", at least one plasma cell, scored the highest false-positive rate.

\section{Discussion}

This study had three key findings: (1) $\geq 3$ PMN and $\geq 1$ PC per hpf as a histologic criteria for the diagnosis of moderate to severe salpingitis, while performing better than the other criteria, appears to have limited utility even more so for cases of mild salpingitis; (2) endometrial specimens were often unevaluable for histopathology, and unevaluable specimens were more likely in subjects with severe salpingitis and HIVinfection, and thus may affect the utility of endometrial histopathology to confirm the clinical diagnosis of PID in similar settings; (3) The PMN response increased with disease severity for HIV-1 seronegative but not HIV-1 seropositive women with salpingitis.

Since Kiviat et al. published their paper, [8] histologic endometritis has been used as a surrogate marker for salpingitis, especially in the study of mild to moderate PID. Even though the criteria for histologic endometritis had never been validated in HIV-1-infected populations, several studies of PID were conducted in high HIV-1 seroprevalence settings $[6,7,9,12,14]$. The results of this study did not validate the Kiviat et al. 1990, criteria for $\geq 5$ PMNs and $\geq 1$ PC for the diagnosis of PID. In the Kiviat et al. cohort, $N$. gonorrhoeae and/or C. trachomatis was found in 49\% of the patient population; in comparison, the cohort in our study had a high HIV-1 prevalence and a combined gonorrhea and/or chlamydia prevalence of $18 \%$ (Table 1).

Similar to another report, only $72 \%$ of endometrial biopsies in our study were evaluable [1]. The increased frequency of unevaluable endometrial biopsies in women with severe salpingitis, likely due to increased endometrial sloughing and presence of pus, and HIV-1 infection further limits the utility 
TABLe 3: Presence and density of polymorphonuclear leucocytes (PMN) and plasma cells (PC) on histopathology of endometrial biopsies in women with mild, moderate, and severe salpingitis at laparoscopy and controls in HIV-seropositive and -seronegative women.

\begin{tabular}{lcccccc}
\hline & \multicolumn{2}{c}{ Mild salpingitis } & \multicolumn{2}{c}{ Moderate/severe Salpingitis } & \multicolumn{2}{c}{ Controls without salpingitis } \\
Rules representing cell/hpf & \multicolumn{2}{c}{$N=32$} & \multicolumn{2}{c}{$N=50$} & \multicolumn{2}{c}{$N=62$} \\
& HIV +/ve & HIV -/ve & HIV +/ve & HIV -/ve & HIV +/ve & HIV -/ve \\
& $(N=7)$ & $(N=25)$ & $(N=23)$ & $(N=27)$ & $(N=45)$ & $(N=12)$ \\
\hline$\geq 3$ PMN and $\geq 1$ PC & $4(57 \%)$ & $4(16 \%)$ & $17(74 \%)$ & $17(63 \%)$ & $3(7 \%)$ & $3(25 \%)$ \\
$\geq 1$ PMN and $\geq 1$ PC & $7(100 \%)$ & $8(32 \%)$ & $19(83 \%)$ & $20(74 \%)$ & $17(38 \%)$ & $7(58 \%)$ \\
$\geq 1$ PMN & $7(100 \%)$ & $17(68 \%)$ & $21(91 \%)$ & $21(78 \%)$ & $32(71 \%)$ & $8(67 \%)$ \\
$\geq 1$ PC & $7(100 \%)$ & $9(36 \%)$ & $21(91 \%)$ & $21(78 \%)$ & $28(62 \%)$ & $8(67 \%)$ \\
\hline
\end{tabular}

of endometrial histopathology as a diagnostic tool for studies of PID in similar populations.

The low sensitivity of histologic endometritis for mild salpingitis amongst women symptomatic for PID was unexpected. Studies of endometritis in populations of asymptomatic women have consistently demonstrated a relatively high prevalence of endometritis $[10,15,16]$ which led authors to describe endometritis as an intermediate infection to PID. Eckert et al. studied HIV-1-infected women presenting to a family planning clinic and found endometritis in $38 \%$ of participants [10]. This is a higher prevalence than what we found in HIV-1-negative women (16\%) and a lower prevalence than what we found in HIV-1seropositive women $(57 \%)$ with mild salpingitis using less stringent criteria for endometritis. Furthermore, a prior laparoscopic study demonstrated salpingitis in the absence of endometritis [7]. An alternative explanation may result from the subjectivity of the laparoscopic criteria for mild salpingitis that leads to misclassification of cases [13].

The distribution of PMNs and PCs in the endometrium of women with salpingitis was affected by HIV-1 serostatus and disease severity. PMNs are only found in the healthy endometrium during menses [17], and form part of the endometrial immune response, they are also the first line immune defense against bacterial infections. The increased density of PMN with severe disease in HIV-1-uninfected but not in HIV-1-infected women with salpingitis is not well understood. Consistent with other studies [7, 9, 10, 14], we found increased PC endometritis with HIV-1 infection. This could represent HIV-1 infection in the genital tract [18]; chronic plasma cell endometritis [11, 14]; or the presence of opportunistic infections. Cherpes et al. reported an association between HSV-2 seropositivity and plasma cell endometritis [19]; notably HSV-2 is extremely prevalent among HIV-1-infected persons (KAIS 2007 [20]. Contrary to these findings, Eckert et al. [10] evaluated 20 endometrial biopsy samples from women with asymptomatic histologic endometritis and failed to detect herpes simplex virus by PCR, and cytomegalovirus was detected equally in women with and without histological endometritis. Mycoplasma genitalium is another potential cause of endometritis [21].

One limitation of this study is that HIV-1-infected controls did not undergo laparoscopic evaluation. Therefore, unlikely we cannot firmly exclude subclinical salpingitis from this population as we can for the HIV-1-seronegative controls. Furthermore, we did not attempt to detect suspected etiologies of endometritis such as bacteria other than $N$. gonorrhoeae and C. trachomatis including M. genitalium [14] and potential etiologies such as cytomegalovirus and herpes simplex virus infection. Such data might help to elucidate the reason for the different findings among HIV-1seropositive and HIV-1-seronegative women with salpingitis in regards to endometrial histopathology.

This study raises some important questions regarding PID and its sequelae. With increased access to highly active antiretroviral therapy (HAART), HIV-1-infected women are living longer. Population data from Uganda [22] plus others [23] have demonstrated reduced fertility in HIV-1-infected women regardless of disease stage. It is plausible that PC endometritis may lead to reduced fertility. Further research is required to determine if women using HAART return to normal fertility or not. Lastly, although endometrial histopathology serves as a reasonable surrogate for salpingitis in HIV-1-uninfected populations, its utility in populations with a high HIV-1 seroprevalence appears to be limited. Discovery of a sensitive and specific biomarker or set of biomarkers for salpingitis could facilitate further research on PID and its sequelae in such settings.

\section{References}

[1] T. Mathews, J. K. G. Mati, and J. N. Fomulu, "A study of infertility in Kenya: results of investigation of the infertile couple in Nairobi," East African Medical Journal, vol. 58, no. 4, pp. 288-297, 1981.

[2] S. M. Walton and J. K.G. Mati, "An evaluation of secondary infertility in Kenya," East African Medical Journal, vol. 53, no. 6, pp. 310-314, 1976.

[3] L. Westrom, R. Joesoef, G. Reynolds, A. Hagdu, and S. E. Thompson, "Pelvic inflammatory disease and fertility: a cohort study of 1,844 women with laparoscopically verified disease and 657 control women with normal laparoscopic results," Sexually Transmitted Diseases, vol. 19, no. 4, pp. 185192, 1992.

[4] L. Westrom, "Incidence, prevalence, and trends of acute pelvic inflammatory disease and its consequences in industrial countries," American Journal of Obstetrics and Gynecology, vol. 138, no. 7, pp. 880-892, 1980.

[5] A. W. Ojwang, V. M. Lema, and S. H. M. Wanjala, "HIV infection among patients with acute pelvic inflammatory 
disease at the Kenyatta National Hospital, Nairobi, Kenya," East African Medical Journal, vol. 70, no. 8, pp. 506-511, 1993.

[6] M. C. Kamenga, K. M. De Cock, M. E. St Louis et al., "The impact of human immunodeficiency virus infection on pelvic inflammatory disease: a case-control study in Abidjan, Ivory Coast," American Journal of Obstetrics and Gynecology, vol. 172, no. 3, pp. 919-925, 1995.

[7] C. R. Cohen, "Effect of human immunodeficiency virus type 1 infection upon acute salpingitis: a laparoscopic study," Journal of Infectious Diseases, vol. 178, no. 5, pp. 1352-1358, 1998.

[8] N. B. Kiviat, P. Wolner-Hanssen, D. A. Eschenbach et al., "Endometrial histopathology in patients with culture-proved upper genital tract infection and laparoscopically diagnosed acute salpingitis," American Journal of Surgical Pathology, vol. 14, no. 2, pp. 167-175, 1990.

[9] E. A. Bukusi, C. R. Cohen, C. E. Stevens et al., "Effects of human immunodeficiency virus 1 infection on microbial origins of pelvic inflammatory disease and on efficacy of ambulatory oral therapy," American Journal of Obstetrics and Gynecology, vol. 181, no. 6, pp. 1374-1381, 1999.

[10] L. O. Eckert, D. H. Watts, S. S. Thwin, N. Kiviat, K. J. Agnew, and D. A. Eschenbach, "Histologic endometritis in asymptomatic human immunodeficiency virus-infected women: characterization and effect of antimicrobial therapy," Obstetrics and Gynecology, vol. 102, no. 5, pp. 962-969, 2003.

[11] J. A. Kerr-Layton, C. A. Stamm, L. S. Peterson, and J. A. McGregor, "Chronic plasma cell endometritis in hysterectomy specimens of HIV-infected women: a retrospective analysis," Infectious Diseases in Obstetrics and Gynecology, vol. 6, no. 4, pp. 186-190, 1998.

[12] N. R. Mugo, J. A. Kiehlbauch, R. Nguti et al., "Effect of human immunodeficiency virus- 1 infection on treatment outcome of acute salpingitis," Obstetrics and Gynecology, vol. 107, no. 4, pp. 807-812, 2006.

[13] L. Jacobson and L. Weström, "Objectivized diagnosis of acute pelvic inflammatory disease. Diagnostic and prognostic value of routine laparoscopy," American Journal of Obstetrics and Gynecology, vol. 105, no. 7, pp. 1088-1098, 1969.

[14] J. S. Coleman, J. Hitti, E. A. Bukusi et al., "Infectious correlates of HIV-1 shedding in the female upper and lower genital tracts," AIDS, vol. 21, no. 6, pp. 755-759, 2007.

[15] A. P. Korn, N. Hessol, N. Padian et al., "Commonly used diagnostic criteria for pelvic inflammatory disease have poor sensitivity for plasma cell endometritis," Sexually Transmitted Diseases, vol. 22, no. 6, pp. 335-341, 1995.

[16] L. O. Eckert, S. E. Hawes, P. K. Wölner-Hanssen et al., "Endometritis: the clinical-pathologic syndrome," American Journal of Obstetrics and Gynecology, vol. 186, no. 4, pp. 690695, 2002.

[17] C. Poropatich, M. Rojas, and S. G. Silverberg, "Polymorphonuclear leukocytes in the endometrium during the normal menstrual cycle," International Journal of Gynecological Pathology, vol. 6, no. 3, pp. 230-234, 1987.

[18] A. L. Howell, R. D. Edkins, S. E. Rier et al., "Human immunodeficiency virus type 1 infection of cells and tissues from the upper and lower human female reproductive tract," Journal of Virology, vol. 71, no. 5, pp. 3498-3506, 1997.

[19] T. L. Cherpes, H. C. Wiesenfeld, M. A. Melan et al., "The associations between pelvic inflammatory disease, Trichomonas vaginalis infection, and positive herpes simplex virus type 2 serology," Sexually Transmitted Diseases, vol. 33, no. 12, pp. 747-752, 2006.
[20] N. R. Mugo, S. Dadabhai, K. Sabin et al., "National seroprevalence of herpes simplex virus type 2 and co-infection with HIV-1: results of the 2007 Kenya AIDS indicator survey," in International AIDS Conference (IAS), 2009, poster no. MOPEB016.

[21] C. R. Cohen, L. E. Manhart, E. A. Bukusi et al., "Association between Mycoplasma genitalium and acute endometritis," The Lancet, vol. 359, no. 9308, pp. 765-766, 2002.

[22] R. H. Gray, M. J. Wawer, D. Serwadda et al., "Population-based study of fertility in women with HIV-1 infection in Uganda," The Lancet, vol. 351, no. 9096, pp. 98-103, 1998.

[23] A. Ross, D. Morgan, R. Lubega, L. M. Carpenter, B. Mayanja, and J. A. G. Whitworth, "Reduced fertility associated with HIV: the contribution of pre-existing subfertility," AIDS, vol. 13, no. 15, pp. 2133-2141, 1999. 


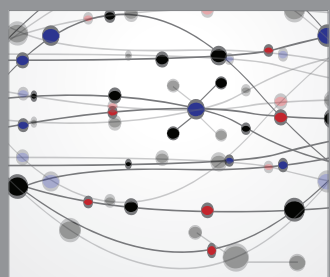

The Scientific World Journal
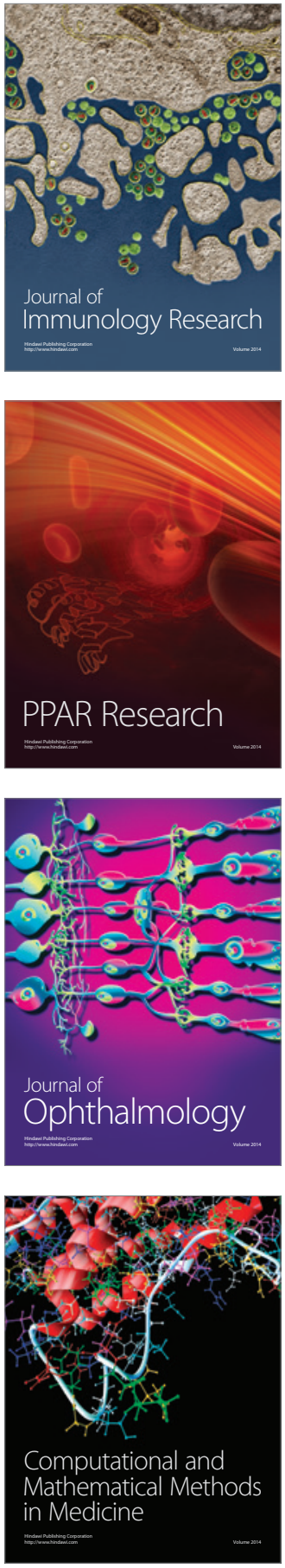

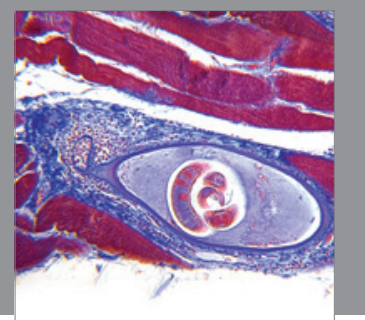

Gastroenterology

Research and Practice
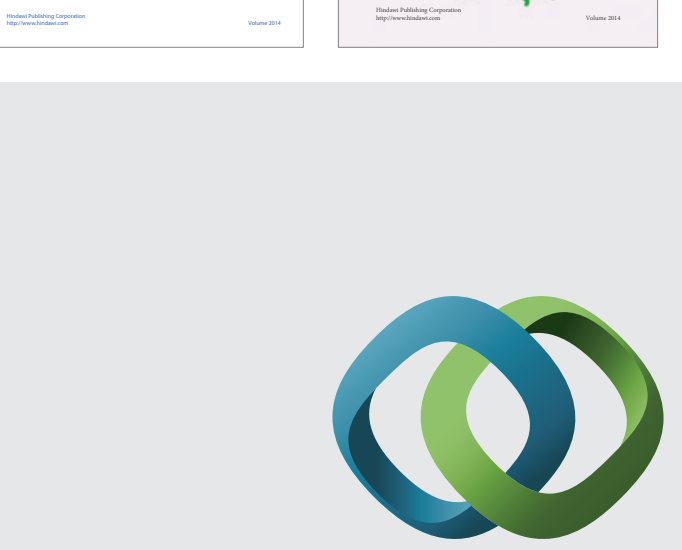

\section{Hindawi}

Submit your manuscripts at

http://www.hindawi.com
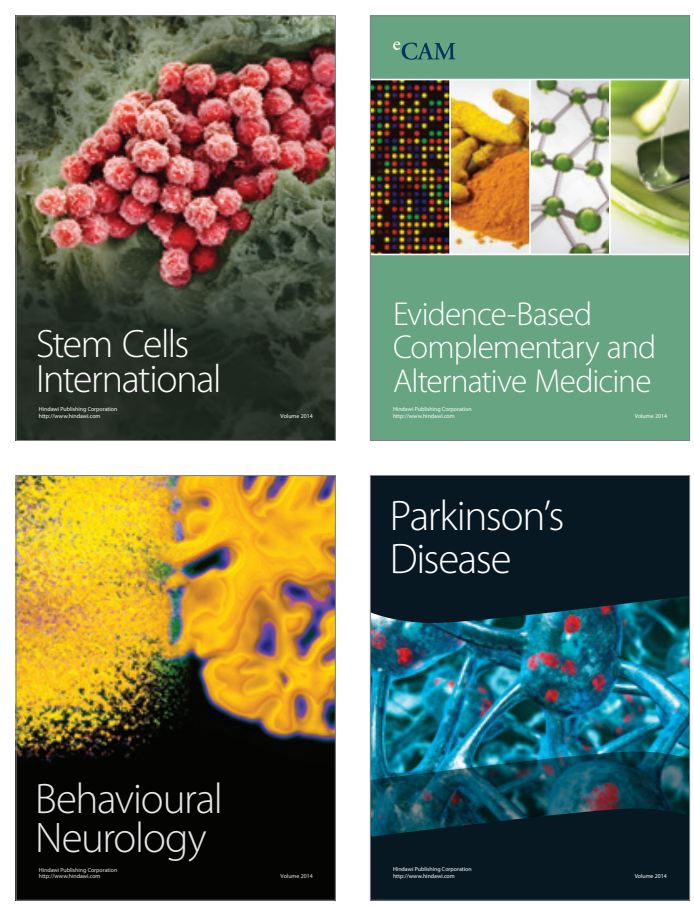

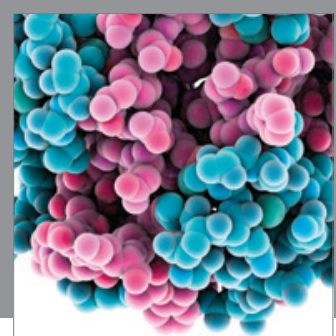

Journal of
Diabetes Research

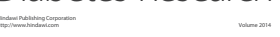

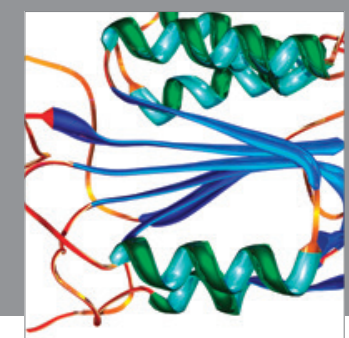

Disease Markers
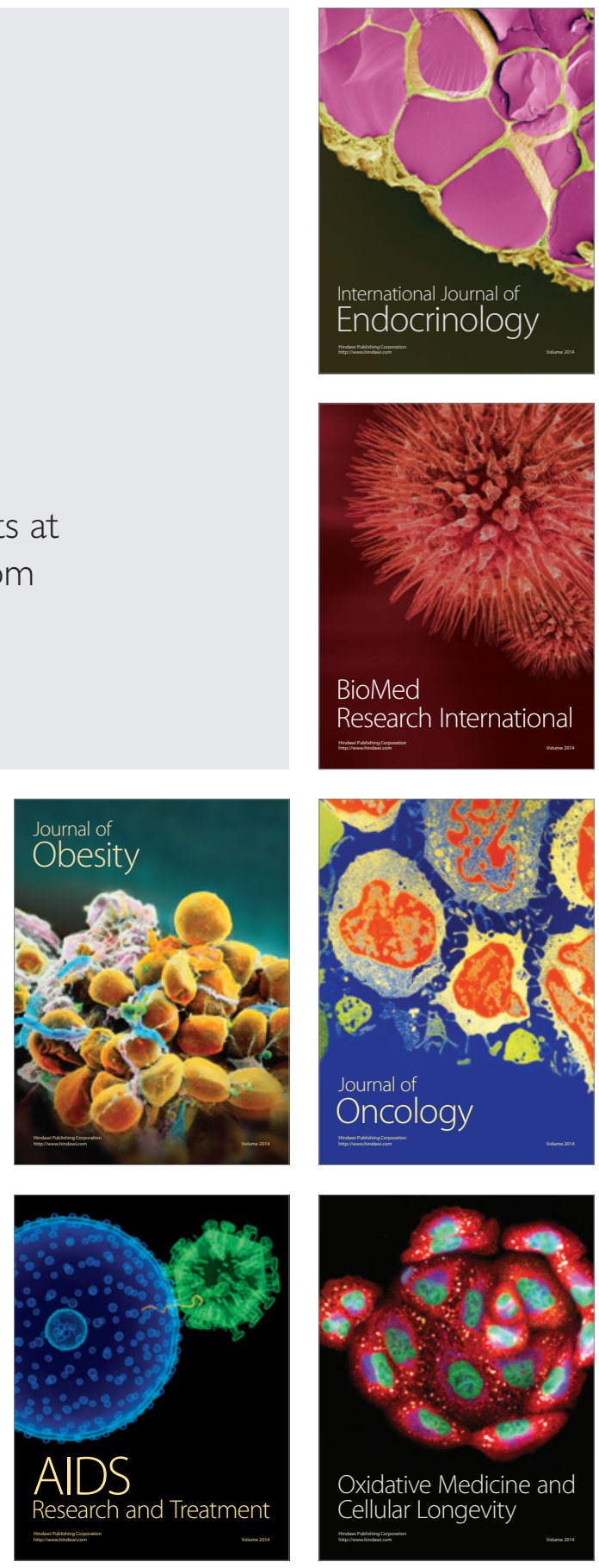